\title{
Communication matters
}

\author{
Is social media a new 4 mat 4 science in UR community? It may be time to sign up.
}

Social media has a reputation for frivolity, and preening your online profile can wreak havoc on productivity. Increasing demands on researchers' time threaten to trigger the hermit instinct even aside from the deluge of new online tools. So why are a growing number of scientists getting on board? Online social networking can raise one's profile in the community, advance research and even help careers, especially for younger scientists seeking to establish themselves. For many, it could be a valuable supplement to more traditional forms of communication.

Broadly speaking, social media refers to the computerenabled exchange of information among groups of individuals. Many scientists are familiar with established platforms such as discussion boards, and most have an online presence on a lab website and a professional networking site such as LinkedIn. But just 13\% of the 3,500 respondents to a recent Nature News survey claimed to use the microblogging service Twitter regularly.

There is justifiable fear that being active online will squander precious time. Compounding this is an unease about whether science fits into the modern trajectory of increasingly fragmented communication. The Twitter format is essentially a public form of texting that limits each message, or 'tweet', to 140 characters. How can this help but reduce science to buzz, sound bites and superficial dressing? Where's the beef?

A form of scientific discourse is possible on Twitter, despite the brevity. At its best, the space constraint demands creativity and skill to distill ideas that are worth broadcasting. A large fraction of scientific tweets link to indepth sources, and online exchanges can become a referral service for interesting blog posts, preliminary data and papers. It also brings speed. Live-tweeting at conferences involves the audience in vibrant real-time discussions. The discussions include colleagues far from the meeting and can carry on throughout the year, what astrophysicist and avid tweeter Katie Mack has called "a kind of ongoing virtual conference coffee break."

Different media have different strengths. For more indepth reflection and context, long-format blogs examine subjects from broad to niche. A key difference is the investment of time and thought that is needed; a tweet demands a much smaller commitment and typically reaches more people than a blog post but is more limited and ephemeral. Broadcasting comments and ideas in either format can bring useful feedback and critique. Likewise, following a diversity of peers can tune you into recent community news and stimulate creativity.
Scientific social networking platforms such as Academia.edu and ResearchGate may make it more likely to be discovered, and social bookmarking and reference management tools enable suggestions and comment sharing on papers.

The Internet paradigm is one of dispersed information in a sea of noise, and mastering filtering techniques (both digital and mental) can greatly improve how well you find gold among the silt. Applications that monitor and aggregate feeds from different services can help. But doing this effectively can require adjustments in the way you work: perhaps by using spare moments to slip into Twitter streams (updates by users or topics that you follow) for breaking news, questions and conversation and by performing systematic blog searches to keep up with a set of subjects. Making these tools productive rather than a distraction from sustained thought takes discipline within science just as it does outside of it.

Social media does involve additional risks. It can allow a few active voices to dominate a community, and it enables mob behavior. Messages can be taken out of context, and mistakes are easily amplified. It is also easy to accidentally expose sensitive information to current or future professional colleagues.

To be sure, the impact of a social presence is difficult to measure objectively. The effect of social media mentions on scholarly citations, for example, is debated. Some studies show no effect; others find a significant effect but cannot distinguish whether social attention brings citations or vice versa. Yet it is safe to say that cultivating interest in your work is rarely a bad thing. Alternative metrics, or 'altmetrics'-such as paper downloads, website visits and mentions on social media-capture dimensions of a paper's influence beyond citations. And informal surveys indicate that although social media engagement is not yet a component of faculty hiring and promotion decisions, its potential contribution to scientific life and public outreach is increasingly appreciated.

There is much to be gained from a judicious use of social media within science: a wider ear to hear what others find interesting, an expanding scientific network, more feedback and discussion on your work, a source of new ideas, and simply more information, faster. Communication and discussion are bedrocks of science, and social media can be an aid to these if handled with care. 